\title{
On Hölder regularity of the singular set of energy minimizing harmonic maps into closed manifolds
}

\author{
Michał Miśkiewicz ${ }^{1}$
}

Received: 10 July 2018 / Accepted: 30 November 2019 / Published online: 20 January 2020

(c) The Author(s) 2020

\begin{abstract}
Energy minimizing harmonic maps between manifolds are known to be smooth outside a rectifiable set of codimension 3 , called the singular set. The possibility that this set is not a manifold, but has arbitrarily many small gaps in it, is not excluded in general. Here we prove that some part of the singular set—characterized by topological and analytic properties of tangent maps-is a topological manifold. In the special case of maps into the sphere $\mathbb{S}^{2}$, we conclude that the whole top-dimensional part of the singular set is a manifold-this generalizes a similar result in four-dimensional domains, due to Hardt and Lin.
\end{abstract}

Mathematics Subject Classification $35 \mathrm{~J} 60 \cdot 53 \mathrm{C} 43 \cdot 58 \mathrm{E} 20$

\section{Introduction}

\subsection{Singularities of Energy Minimizing Harmonic Maps}

Energy minimizing harmonic maps between manifolds may have singularities if the domain dimension is 3 or higher. The most well-known example is the map

$$
\mathbb{R}^{3} \times \mathbb{R}^{n-3} \ni(x, y) \mapsto x /|x| \in \mathbb{S}^{2} .
$$

In general, any energy minimizer $u$ is smooth outside the closed singular set $\operatorname{sing} u$ of Hausdorff dimension $n-3$ or less, $n$ being the dimension of the domain (Schoen, Uhlenbeck $[11,13])$. The phenomenon of singularities is now well-understood in dimension 3 , when singularities form a discrete set. In recent years, there has been a substantial progress concerning the case $n \geqslant 4$. Naber and Valtorta [7] have proved that the singular set has locally finite

Communicated by F. H. Lin.

The research has been supported by the NCN Grants no. 2016/21/B/ST1/03138 (years 2017-2020) 2018/28/T/ST1/00117.

$凶 \quad$ Michał Miśkiewicz

m.miskiewicz@mimuw.edu.pl

1 Institute of Mathematics, University of Warsaw, Banacha 2, 02-097 Warsaw, Poland 
$(n-3)$-dimensional Hausdorff measure and is $(n-3)$-rectifiable, i.e., can be essentially covered by countably many Lipschitz images of $\mathbb{R}^{n-3}$; the latter was already known (due to Simon [16]) in the case when the target manifold is real-analytic.

The results cited above are mostly concerned with the size of the singular set, but do not imply lower bounds on the singular set. In particular, the possibility that the singular set is an arbitrary subset of an $(n-3)$-dimensional manifold (with many small gaps) is not excluded by $[7,11,13,16]$.

Lower bounds on the size are indeed possible in the presence of a topological obstruction; the following example is simple but instructive.

Example 1.1 Consider the smooth boundary map $\varphi: \mathbb{S}^{2} \times \mathbb{S}^{1} \rightarrow \mathbb{S}^{2}$ given by $\varphi(x, y)=x$ and (some) $u: \mathbf{B}^{3} \times \mathbb{S}^{1} \rightarrow \mathbb{S}^{2}$ minimizing the energy in the class of maps equal to $\varphi$ on the boundary. Restricting $u$ to a slice $\mathbf{B}^{3} \times\{y\}$ and applying Brouwer's theorem, we see that each such slice contains a singular point. This shows that $\mathcal{H}^{1}(\operatorname{sing} u) \geqslant \mathcal{H}^{1}\left(\mathbb{S}^{1}\right)=2 \pi$. In this particular case one can actually prove that $u(x, y)=x /|x|$, but the presented reasoning applies also to every $\varphi^{\prime}: \mathbb{S}^{2} \times \mathbb{S}^{1} \rightarrow \mathbb{S}^{2}$ homotopic to $\varphi$.

In the special case of maps $u: \mathbf{B}^{4} \rightarrow \mathbb{S}^{2}$, Hardt and Lin [2] obtained the following remarkable result.

Theorem 1.2 The singular set of an energy minimizer $u: \mathbf{B}^{4} \rightarrow \mathbb{S}^{2}$ is locally a union of a finite set and a finite family of Hölder continuous closed curves with a finite number of crossings.

The same claim was obtained also for maps $u: \mathbf{B}^{5} \rightarrow \mathbb{S}^{3}$ (Lin-Wang [3]). To the author's knowledge, these are the only two cases where $\operatorname{sing} u$ was shown to be essentially a manifold.

The above theorem relies on the classification of tangent maps from $\mathbb{R}^{3}$ into $\mathbb{S}^{2}$ carried out by Brezis et al. [1]; for $\mathbb{S}^{3}$, a similar classification was obtained by Nakajima [8]. These maps describe the infinitesimal behavior of $u$ at a typical point of $\operatorname{sing} u$.

\subsection{Main Results}

The present paper aims to extract the topological obstruction responsible for preventing gaps in the singular set of maps into $\mathbb{S}^{2}$. To this end, we distinguish particular homotopy classes of tangent maps $\mathbb{R}^{3} \rightarrow \mathcal{N}$ (called here indecomposable classes) for any closed Riemannian manifold $\mathcal{N}$.

To each homotopy class $\alpha \in \pi_{2}(\mathcal{N})$ we assign its lowest energy level $\Theta(\alpha)$ and call $\alpha$ indecomposable if $\Theta(\alpha)<\infty$ and $\alpha$ cannot be represented as a sum of homotopy classes $\alpha_{j} \in \pi_{2}(\mathcal{N})$ with strictly smaller energy levels $\Theta\left(\alpha_{j}\right)$. We then restrict our attention to singularites with fixed topological type $\alpha$-we define $\operatorname{sing}_{\alpha} u$ to be the set of points at which some tangent map of $u$ has type $\alpha$. Rigorous definitions are given in Sect.2.3.

Another goal is to generalize the result of Hardt and Lin [2] to higher dimensional domains. The difficulty lies in the fact that the singular set is stratified-it decomposes into parts of different dimensions. For $u: \mathbf{B}^{4} \rightarrow \mathbb{S}^{2}$, there are only two strata: one is formed by Hölder continuous curves and the other by their crossing points and a finite number of additional isolated points. In the theorem below, we were only able to study the top-dimensional part $\operatorname{sing}_{*} u$ of the singular set. Again, the necessary notions are introduced in Sect. 2.2.

For simplicity, we only consider the standard Euclidean ball $\mathbf{B}^{n}$ as the domain, but the results hold true for any manifold. This is due to the fact that we only consider the infinitesimal behavior of maps. A detailed explanation can be found in [7] and [16, Sect. 8]. 
Theorem 1.3 Let $u: \mathbf{B}^{n} \rightarrow \mathcal{N}$ be an energy minimizing map into a closed Riemannian manifold $\mathcal{N}, \alpha \in \pi_{2}(\mathcal{N})$ be an indecomposable homotopy class, and $\Theta(\alpha)$ be its lowest energy level. Then for each exponent $0<\gamma<1$ there is $\delta(\gamma, n, \alpha, \mathcal{N})>0$ such that the set

$$
\left\{x \in \operatorname{sing}_{\alpha} u: \lim _{r \rightarrow 0} r^{2-n} \int_{\mathbf{B}_{r}(x)}|\nabla u|^{2}<\Theta(\alpha)+\delta\right\}
$$

forms an open subset of $\operatorname{sing} u$ and it is a topological $(n-3)$-dimensional manifold of Hölder class $C^{0, \gamma}$.

In the case when $\mathcal{N}$ is a real-analytic manifold, Simon [16, Lemma 4.3] showed that the set of possible energy densities $\lim _{r \rightarrow 0} r^{2-n} \int_{\mathbf{B}_{r}(x)}|\nabla u|^{2}$ is discrete. This allows us to slightly strenghten the statement above. The same conclusion holds also if $\mathcal{N}$ satisfies the integrability assumption introduced in [17, Ch. 3.13].

Corollary 1.4 If $u: \mathbf{B}^{n} \rightarrow \mathcal{N}$ is an energy minimizing map into a real-analytic manifold $\mathcal{N}$ and $\alpha \in \pi_{2}(\mathcal{N})$ is an indecomposable homotopy class, then

$$
\left\{x \in \operatorname{sing}_{\alpha} u: \lim _{r \rightarrow 0} r^{2-n} \int_{\mathbf{B}_{r}(x)}|\nabla u|^{2}=\Theta(\alpha)\right\}
$$

forms an open subset of $\operatorname{sing} u$ and it is a topological $(n-3)$-dimensional manifold of Hölder class $C^{0, \gamma}$ with any $0<\gamma<1$.

Specializing to the case $\mathcal{N}=\mathbb{S}^{2}$ and recalling the classification of tangent maps [1], we obtain a partial generalization of Theorem 1.2 [2] to arbitrary dimensions:

Corollary 1.5 If $u: \mathbf{B}^{n} \rightarrow \mathbb{S}^{2}$ is an energy minimizing map, then the top-dimensional part $\operatorname{sing}_{*} u$ forms an open subset of $\operatorname{sing} u$ and it is a topological $(n-3)$-dimensional manifold of Hölder class $C^{0, \gamma}$ with any $0<\gamma<1$.

\subsection{Higher Regularity}

The regularity obtained here for the singular set follows from the classical Reifenberg theorem [9]; this is why our methods are limited to Hölder regularity with any exponent smaller than 1. On the other hand, Naber and Valtorta [7] showed rectifiability of the singular set and it seems possible that further improvements of their Reifenberg-type results (together with the additional topological information derived here) could lead to Lipschitz regularity of the singular set.

Moreover, in his paper on singularities of minimal surfaces [15], Simon remarked that a similar strategy could be applied to harmonic maps and possibly establish $C^{1, \alpha}$ regularity for the singular set.

\subsection{An Outline}

Section 2 recalls all notions and results needed in the sequel. These mostly come from the seminal work of Schoen and Uhlenbeck [11], but the presentation here follows Simon's lecture notes [17]. We also define indecomposable homotopy classes of maps from $\mathbb{S}^{2}$ into $\mathcal{N}$.

Since Theorem 1.3 only concerns the singularities of indecomposable types, it is worthwhile to investigate the existence of such classes, which we do in Sect. 3. Indeed, we show that 
for any $\mathcal{N}$ the second homotopy group $\pi_{2}(\mathcal{N}, p)$ is generated (up to the action of $\pi_{1}(\mathcal{N}, p)$ on $\left.\pi_{2}(\mathcal{N}, p)\right)$ by indecomposable homotopy classes. This is very close to the classical (slightly weaker) result due to Sacks and Uhlenbeck [10] (see also [18]): smooth harmonic maps from $\mathbb{S}^{2}$ into $\mathcal{N}$ generate the whole group $\pi_{2}(\mathcal{N}, p)$ (up to the action of $\pi_{1}(\mathcal{N}, p)$ ).

To obtain bi-Hölder-equivalence with a Euclidean ball, we employ Reifenberg's topological disc theorem [9] (see also [14]). We recall its statement and the so-called Reifenberg flatness condition in Sect. 4. We also introduce a flatness condition for an energy minimizer $u$ which includes Reifenberg flatness for $\operatorname{sing} u$, but also forces $u$ to be close to a tangent map.

The main results are proved in Sect.5. The difficulty in applying Reifenberg's theorem to sing $u$ lies in showing that this set has no gaps. This is done in Lemma 5.1; this is also the point where our topological assumptions play a role. Then we are able to show that if $u$ satisfies the flatness condition on the ball $\mathbf{B}_{2}(0)$, it also satisfies the same condition on each smaller ball $\mathbf{B}_{r}(0)$ (Corollary 5.5) and on each ball $\mathbf{B}_{1}(z)$ centered at a point $z \in \mathbf{B}_{1}$ with enough energy density (Proposition 5.7). Combining these results, we check the hypotheses of Reifenberg's theorem and establish Theorem 1.3.

Some interesting observations not needed for the proof of Theorem 1.3 are gathered in Sect. 6.

\section{Preliminaries}

\subsection{Regularity of Energy Minimizers}

In what follows, $u: \mathbf{B}^{n} \rightarrow \mathcal{N}$ is an energy minimizing map into a closed Riemannian manifold $\mathcal{N}$. Here we recall the basic properties of such maps [11].

Denote the rescaled energy

$$
\theta_{u}(x, r):=r^{2-n} \int_{\mathbf{B}_{r}(x)}|\nabla u|^{2} \text { for } \mathbf{B}_{r}(x) \subseteq \mathbf{B}_{1},
$$

which is monotone in $r$ :

$$
\frac{\partial}{\partial r} \theta_{u}(x, r)=2 \int_{\partial \mathbf{B}_{r}(x)} \frac{|\nabla u \cdot(y-x)|^{2}}{|y-x|^{n}} \geqslant 0 .
$$

This enables us to define the energy density at $x$ :

$$
\theta_{u}(x, 0):=\lim _{r \rightarrow 0} \theta_{u}(x, r),
$$

which is by definition an upper semicontinuous function (in both $x \in \mathbf{B}^{n}$ and $u \in W^{1,2}$ ) [17, 2.11]. Obviously, $\theta_{u}(x, 0)=0$ at regular points.

The main regularity statement is the following $\varepsilon$-regularity theorem:

$$
\begin{aligned}
& \text { there is } \varepsilon(n, \mathcal{N})>0 \text { s.t. } \theta_{u}(x, 2 r)<\varepsilon \Rightarrow u \text { is smooth on } \mathbf{B}_{r}(x), \\
& \quad \text { in particular } \theta_{u}(x, 0)<\varepsilon \Rightarrow x \notin \operatorname{sing} u .
\end{aligned}
$$

We also note two compactness theorems for a sequence $u_{k}$ of energy minimizers:

- If $u_{k} \rightarrow u$ in $W^{1,2}(\Omega)$, then $u$ is an energy minimizer and the convergence is actually strong in $W_{\text {loc }}^{1,2}(\Omega)[4]$,

- If $u_{k} \rightarrow u$ as above, then the convergence is uniform on compact sets disjoint from $\operatorname{sing} u$ [11]. 


\subsection{Tangent Maps}

Given an energy minimizer $u: \mathbf{B}^{n} \rightarrow \mathcal{N}$ and a point $x \in \mathbf{B}^{n}$, consider the family of rescaled maps $u_{r}(y)=u(x+r y)$. By the results from the previous section, each sequence $r_{j} \rightarrow 0$ has a subsequence for which $u_{r_{j}}$ converges in $W_{\text {loc }}^{1,2}\left(\mathbb{R}^{n}\right)$ to some energy minimizer $\varphi$, called a tangent map of $u$ at $x$ (possibly non-unique). This limit map is homogeneous, i.e., $\varphi(\lambda x)=$ $\varphi(x)$ for all $\lambda>0, x \in \mathbb{R}^{n}$.

For a homogeneous energy minimizer $\varphi: \mathbb{R}^{n} \rightarrow \mathcal{N}$, the energy density $\theta_{\varphi}(y, 0)$ is maximal at $y=0$; moreover, equality $\theta_{\varphi}(y)=\theta_{\varphi}(0)$ at some other point $y$ leads to higher symmetry: $\varphi(x+t y)=\varphi(x)$ for all $t \in \mathbb{R}, x \in \mathbb{R}^{n}$. Let $S(\varphi)$ be defined by

$$
S(\varphi)=\left\{y \in \mathbb{R}^{n}: \theta_{\varphi}(y)=\theta_{\varphi}(0)\right\} .
$$

Then $S(\varphi)$ is a linear subspace of $\mathbb{R}^{n}$ describing the symmetries of $\varphi$ :

$$
\varphi(x+y)=\varphi(x) \text { for all } x \in \mathbb{R}^{n}, y \in S(\varphi) .
$$

For non-constant $\varphi$, we have $S(\varphi) \subseteq \operatorname{sing} \varphi$. If $\operatorname{dim} S(\varphi)=n-3$, this is necessarily an equality.

\subsection{Top-Dimensional Part of the Singular Set}

If $u$ is an energy minimizer, for each $j=0,1,2, \ldots, n-1$ we define

$$
S_{j}=\{y \in \operatorname{sing} u: \operatorname{dim} S(\varphi) \leqslant j \text { for all tangent maps } \varphi \text { of } u \text { at } y\},
$$

which leads to the classical stratification of the singular set

$$
S_{0} \subseteq S_{1} \subseteq \cdots \subseteq S_{n-3}=S_{n-2}=S_{n-1}=\operatorname{sing} u .
$$

It is known [11] that each $S_{j}$ has Hausdorff dimension at most $j$, in particular

$$
\operatorname{dim}_{H} \operatorname{sing} u \leqslant n-3 .
$$

In what follows, we are interested in the top-dimensional part of the singular set:

$$
\begin{aligned}
\operatorname{sing}_{*} u & =S_{n-3} \backslash S_{n-4} \\
& =\{y \in \operatorname{sing} u: \operatorname{dim} S(\varphi)=n-3 \text { for some tangent map } \varphi \text { of } u \text { at } y\}
\end{aligned}
$$

Note that

$$
\operatorname{dim}_{H}\left(\operatorname{sing} u \backslash \operatorname{sing}_{*} u\right) \leqslant n-4 .
$$

Following [17], we shall call any homogeneous energy minimizing $\varphi: \mathbb{R}^{n} \rightarrow \mathcal{N}$ with $\operatorname{dim} S(\varphi)=n-3$ a homogeneous cylindrical map (abbreviated HCM).

Consider now such a $\operatorname{HCM} \varphi_{0}: \mathbb{R}^{n} \rightarrow \mathcal{N}$ with $S\left(\varphi_{0}\right)=\mathbb{R}^{n-3} \times \mathbf{0}$. This map actually depends only on 3 variables, i.e. $\varphi_{0}(x, y)=\varphi_{1}(y)$ for some homogeneous $\varphi_{1}: \mathbb{R}^{3} \rightarrow \mathcal{N}$. By [2, Lemma 2.1], the map $\varphi_{1}$ defined in this way is energy minimizing if and only if $\varphi_{0}$ is. Since $\varphi_{1}$ is homogeneous, it is uniquely determined by its restriction to the unit sphere $\varphi_{2}: \mathbb{S}^{2} \rightarrow \mathcal{N}$, which is a smooth harmonic map.

From now on, we shall abuse the notation and use the same symbol for all three maps $\varphi_{0}, \varphi_{1}, \varphi_{2}$; the precise meaning should be clear from the context. Note that their energies 
differ by a multiplicative constant:

$$
\int_{\mathbb{S}^{2}}\left|\nabla \varphi_{2}\right|^{2}=\int_{\mathbf{B}_{1}^{3}}\left|\nabla \varphi_{1}\right|^{2}=C(n) \int_{\mathbf{B}_{1}^{n}}\left|\nabla \varphi_{0}\right|^{2},
$$

so energy comparison does not lead to confusion.

Homotopy type of a HCM always refers to the map $\varphi_{2}: \mathbb{S}^{2} \rightarrow \mathcal{N}$ (as $\varphi_{0}, \varphi_{1}$ are discontinuous and defined on contractible domains). For a general $\operatorname{HCM} \varphi_{0}$ we may choose a rotation $q$ that maps $S\left(\varphi_{0}\right)$ to $\mathbb{R}^{n-3} \times \mathbf{0}$ and thus reduce to the previous case. We then say that $\varphi_{0}$ has homotopy type $\alpha$ if $\varphi_{0} \circ q^{-1}$ restricted to $\mathbf{0} \times \mathbb{S}^{2}$ has type $\alpha$.

Remark 2.1 There is a subtle ambiguity here. Depending on the choice of $q$, we may obtain two homotopy types that differ by a composition with the antipodal map, i.e. both $\left[\varphi_{2}(x)\right]$ and $\left[\varphi_{2}(-x)\right]$.

Using this terminology, singular points in $\operatorname{sing}_{*} u$ can be classified according to their energy density and the homotopy type of a tangent map. Since we only consider basepointfree homotopies, we denote by $\pi_{2}(\mathcal{N})$ the set of homotopy classes of continuous maps $\mathbb{S}^{2} \rightarrow \mathcal{N}$.

Remark 2.1 To avoid confusion, we distinguish the set of basepoint-free homotopy classes $\pi_{2}(\mathcal{N})$ from the second fundamental group $\pi_{2}(\mathcal{N}, p)$. Note that in general $\pi_{2}(\mathcal{N})$ does not carry a group structure, as it is the quotient of the action of $\pi_{1}(\mathcal{N}, p)$ on $\pi_{2}(\mathcal{N}, p)$.

Definition 2.2 For any homotopy type $\alpha \in \pi_{2}(\mathcal{N})$ we let

$\operatorname{sing}_{\alpha} u=\{y \in \operatorname{sing} u$ : some tangent map of $u$ at $y$ is a HCM of type $\alpha\}$.

We also denote its lowest energy level by

$$
\Theta(\alpha):=\inf \left\{\int_{\mathbf{B}_{1}^{n}}|\nabla \varphi|^{2}: \varphi \text { is a HCM of type } \alpha\right\} .
$$

A simple compactness argument shows that this infimum is either infinite (if no HCM has type $\alpha$ ) or achieved by some minimal HCM. We also let

$$
\operatorname{sing}_{\geqslant \Theta} u=\left\{y \in \operatorname{sing} u: \theta_{u}(y, 0) \geqslant \Theta\right\} .
$$

which is a closed set by upper semicontinuity of $\theta_{u}(\cdot, 0)$.

At this point we cannot exclude the case when there are many homotopically different tangent maps at one point. However, this cannot happen under an additional assumption described below (see Remark 5.10). Again, the decomposition in Definition 2.3 is to be understood up to the action of $\pi_{1}(\mathcal{N})$, as described in Sect. 3 (see also the formulation of [10, Thm. 5.9]).

Definition 2.3 Consider $\alpha \in \pi_{2}(\mathcal{N})$ with $\Theta(\alpha)<\infty$. This homotopy class is called decomposable if there is a decomposition

$$
\alpha=\alpha_{1}+\cdots+\alpha_{k} \text { in } \pi_{2}(\mathcal{N}),
$$

where $\Theta\left(\alpha_{j}\right)<\Theta(\alpha)$ for each $j=1, \ldots, k$. Otherwise $\alpha$ is called indecomposable. 
Note that the above criterion does not depend on the dimension $n$, but only on the manifold $\mathcal{N}$.

As a special case, $\alpha$ is indecomposable if $\Theta(\alpha)$ is the smallest among all non-trivial homotopy types. In this case the proof is much easier (see the remark below Lemma 5.1).

Remark 2.2 Similar decompositions of this type appear naturally as a result of the bubbling phenomenon when one tries to minimize the energy in a given homotopy class. More precisely, recall that by [10] (see also [18]) any smooth map $\varphi: \mathbb{S}^{2} \rightarrow \mathcal{N}$ can be decomposed as $[\varphi]=\left[\varphi_{1}\right]+\cdots+\left[\varphi_{k}\right]$, where each $\varphi_{j}$ is a harmonic map and

$$
\sum_{j=1}^{k} \int_{\mathbb{S}^{2}}\left|\nabla \varphi_{j}\right|^{2} \leqslant \int_{\mathbb{S}^{2}}|\nabla \varphi|^{2} .
$$

Motivated by these decompositions, one could replace the condition $\Theta(\alpha)>\max _{j} \Theta\left(\alpha_{j}\right)$ in Definition 2.3 by $\Theta(\alpha) \geqslant \sum_{j} \Theta\left(\alpha_{j}\right)$, thus enlarging the set of indecomposable classes. A natural conjecture here would be that Theorem 1.3 continues to hold in this case, but the author was not able to verify it.

Example 2.4 By the classification from [1], the only HCMs into the sphere $\mathbb{S}^{2}$ are isometries $\varphi: \mathbb{S}^{2} \rightarrow \mathbb{S}^{2}$. Thus, for $\alpha \in \pi_{2}\left(\mathbb{S}^{2}\right)$ we have

$$
\Theta(\alpha)= \begin{cases}0 & \text { for } \alpha=0 \\ 4 \pi & \text { for } \alpha=[ \pm \mathrm{id}] \\ \infty & \text { otherwise. }\end{cases}
$$

By Definition 2.3, the indecomposable classes here are 0, [id], [-id]. Note that these classes generate the whole group $\pi_{2}\left(\mathbb{S}^{2}\right)$ (see Proposition 3.1 for the general case).

\section{Existence of Indecomposable Homotopy Classes}

We show that the set of all indecomposable homotopy classes generates $\pi_{2}(\mathcal{N})$. Similarly to [10, Thm. 5.9], we only consider basepoint-free homotopies, so this statement should be understood as generating $\pi_{2}(\mathcal{N}, p)$ up to the action of $\pi_{1}(\mathcal{N}, p)$. In other words, for any $\alpha \in \pi_{2}(\mathcal{N})$ there are indecomposable homotopy classes $\alpha_{1}, \ldots, \alpha_{k} \in \pi_{2}(\mathcal{N})$ and a continuous map

$$
u: \mathbf{B}^{3} \backslash \bigcup_{j=1}^{k} \mathbf{B}_{j} \rightarrow \mathcal{N}
$$

such that $\left.u\right|_{\partial \mathbf{B}} \in \alpha$ and $\left.u\right|_{\partial \mathbf{B}_{j}} \in \alpha_{j}$, where $\mathbf{B}_{j} \Subset \mathbf{B}$ are smaller disjoint balls.

This can be divided into two steps as follows. The first one is due to Schoen and Uhlenbeck [12, Prop. 3.3].

Proposition 3.1 Let $\mathcal{N}$ be a closed Riemannian manifold. Then

(a) The set of all HCMs $\varphi: \mathbb{S}^{2} \rightarrow \mathcal{N}$ generates $\pi_{2}(\mathcal{N})$,

(b) Each HCM $\varphi: \mathbb{S}^{2} \rightarrow \mathcal{N}$ as an element of $\pi_{2}(\mathcal{N})$ can be decomposed into indecomposable homotopy classes. 
Proof To show part (a), fix $\alpha \in \pi_{2}(\mathcal{N})$ and choose a smooth map $\varphi: \mathbb{S}^{2} \rightarrow \mathcal{N}$ of this type. Then there exists (possibly non-unique) $u \in W^{1,2}\left(\mathbf{B}_{1}^{3}, \mathcal{N}\right)$ such that

$$
\int_{\mathbf{B}_{1}}|\nabla u|^{2}=\min \left\{\int_{\mathbf{B}_{1}}|\nabla v|^{2}: v \in W^{1,2}\left(\mathbf{B}_{1}, \mathcal{N}\right), v=\varphi \text { on } \mathbb{S}^{2}\right\} .
$$

Such a minimizer has at most a finite number of interior singularities $p_{1}, \ldots, p_{k} \in \mathbf{B}_{1}$. At each $p_{j}$ there is a (possibly non-unique) tangent map $\varphi_{j}$, which is necessarily a HCM; by uniform convergence away from the singularity, $u$ restricted to $\partial \mathbf{B}_{r}\left(p_{j}\right)$ is homotopic to $\varphi_{j}$ for some arbitrary small $r$ (in consequence, also for all sufficiently small $r$ ). This yields the decomposition

$$
[\varphi]=\left[\varphi_{1}\right]+\cdots+\left[\varphi_{k}\right] \quad \text { in } \pi_{2}(\mathcal{N}) .
$$

Part (b) follows from the definition by a compactness argument, which allows us to exclude infinite decompositions. Consider any homotopy type $\alpha \in \mathcal{N}$ represented by a HCM, i.e. with $\Theta(\alpha)<\infty$. First let us show that there are only finitely many homotopy types $\beta \in$ $\pi_{2}(\mathcal{N})$ with $\Theta(\beta) \leqslant \Theta(\alpha)$. Indeed, otherwise we would have an infinite sequence of HCMs $\varphi_{k}: \mathbf{B}_{1}^{3} \rightarrow \mathcal{N}$ with distinct homotopy types and uniformly bounded energy. Without loss of generality, $\varphi_{k}$ converges to some $\operatorname{HCM} \varphi$ in $W^{1,2}\left(\mathbf{B}_{1}\right)$, but also in $C^{0}\left(\mathbb{S}^{2}\right)$. This shows that almost all $\varphi_{k}$ have the same homotopy type as $\varphi$, which is a contradiction.

If $\alpha$ is decomposable, we have $\alpha=\alpha_{1}+\cdots+\alpha_{k}$, where $\Theta\left(\alpha_{j}\right)<\Theta(\alpha)$ for each $j$. Decomposing further each $\alpha_{j}$ whenever possible, and iterating this procedure until all obtained homotopy types are indecomposable, we arrive at claim (b). One only needs to note that this procedure stops after at most $N$ steps, where $N$ is the number of homotopy types from the last paragraph. Indeed, any branch of the decomposition tree is a sequence $\beta_{0}, \beta_{1}, \beta_{2}, \ldots$ with $\beta_{0}=\alpha$ and $\Theta\left(\beta_{j+1}\right)<\Theta\left(\beta_{j}\right)$ for each $j$, so it contains at most $N$ elements.

We remark that a similar decomposition was first obtained by Sacks and Uhlenbeck [10] (see also [18]): smooth harmonic maps from $\mathbb{S}^{2}$ into $\mathcal{N}$ generate the whole group $\pi_{2}(\mathcal{N}, p)$ up to the action of $\pi_{1}(\mathcal{N}, p)$. It may be that some homotopy classes in $\pi_{2}(\mathcal{N})$ do not contain any harmonic map. Since here we only consider harmonic maps $\varphi: \mathbb{S}^{2} \rightarrow \mathcal{N}$ for which the homogeneous extension $\varphi: \mathbf{B}^{3} \rightarrow \mathcal{N}$ is energy minimizing, the result discussed above is slightly more general.

\section{Notions of Flatness and Reifenberg's Topological Disc Theorem}

Hölder regularity of the singular set will be obtained by an application of Reifenberg's topological disc theorem [9] (see also [14]). To state it, we first need the following notion of flatness (for our purposes restricted to codimension 3).

Definition 4.1 A set $A \subseteq \mathbb{R}^{n}$ is said to be $\varepsilon$-Reifenberg flat in the ball $\mathbf{B}_{r}(x)$ (with respect to $L)$ if

$$
A \cap \mathbf{B}_{r}(x) \subseteq \mathbf{B}_{r \varepsilon} L \text { and } L \cap \mathbf{B}_{r}(x) \subseteq \mathbf{B}_{r \varepsilon} A
$$

for some $(n-3)$-dimensional affine plane $L$ through $x$.

The above condition means exactly that the normalized Hausdorff distance on $\mathbf{B}_{r}(x)$ from $A$ to some $(n-3)$-dimensional affine plane through $x$ is not larger than $\varepsilon$. 
Theorem 4.2 (Reifenberg's topological disc theorem) For each Hölder exponent $0<\gamma<1$ there is $\varepsilon(n, \gamma)>0$ such that the following holds. If a closed set $A \subseteq \mathbb{R}^{n}$ containing the origin is $\varepsilon$-Reifenberg flat in each ball $\mathbf{B}_{r}(x)$ with $x \in A \cap \mathbf{B}_{1}$ and $r<1$, then the set $A \cap \mathbf{B}_{1}$ is bi-Hölder equivalent to the closed unit ball $\mathbf{B}^{n-3} \subseteq \mathbb{R}^{n-3}$ with exponent $\gamma$.

We shall also make repeated use of the following condition for energy minimizing maps.

Definition 4.3 Fix an indecomposable homotopy class $\alpha \in \pi_{2}(\mathcal{N})$ and let $\Theta=\Theta(\alpha)$ be its lowest energy level (as in Definition 2.2). We say that an energy minimizer $u$ is $\delta$-flat in the ball $\mathbf{B}_{r}(x)$ (of type $\alpha$ ) if

1. $x$ is a singular point of $u$ and $\Theta \leqslant \theta_{u}(x, 0) \leqslant \theta_{u}(x, r) \leqslant \Theta+\delta$,

2. $\operatorname{sing} u$ is $\frac{1}{10}$-Reifenberg flat in $\mathbf{B}_{r}(x)$ with respect to some $L$, and $u$ restricted to the sphere $\left(x+L^{\perp}\right) \cap \partial \mathbf{B}_{r / 2}(x)$ has homotopy type $\alpha$.

From now on, we consider a non-trivial indecomposable class $\alpha$ and its lowest energy level $\Theta=\Theta(\alpha)$ to be fixed.

The main feature of Definition 4.3 is that $\delta$-flatness in a ball trivially ensures that Condition 1 is satisfied in all smaller concentric balls, and one only needs to check Condition 2 (see Corollary 5.5). This is why the constant $\frac{1}{10}$ in Condition 2 was chosen independently of $\delta$.

In fact, one could relax the $\frac{1}{10}$-Reifenberg condition in 2 to $\operatorname{sing} u \cap \mathbf{B}_{r}(x) \subseteq \mathbf{B}_{r / 10} L$. This gives effectively the same notion of $\delta$-flatness, as used in [6].

\section{Regularity of the Singular Set}

\subsection{Persistence of Indecomposable Singularities}

As a first step, we show that if an energy minimizer $u$ restricted to some sphere has homotopy type $\alpha$, then $\operatorname{sing} u$ satisfies the flatness condition of Definition 4.3 and the energy density of $u$ cannot drop in a smaller ball. Note that the claim of Lemma 5.1 is essentially stronger than the condition $L \cap \mathbf{B}_{1} \subseteq \mathbf{B}_{\varepsilon}\left(\operatorname{sing}_{\geqslant \Theta} u\right)$ from Definition 4.1.

Observe that some tubular neighborhood $\mathbf{B}_{\eta} \mathcal{N} \subseteq \mathbb{R}^{M}$ admits a continuous retraction $\pi_{\mathcal{N}}$ onto $\mathcal{N}$. As a consequence, if two continuous functions $f, g$ into $\mathcal{N} \subseteq \mathbb{R}^{M}$ are close enough in supremum norm, then

$$
(t, x) \mapsto \pi_{\mathcal{N}}(t f(x)+(1-t) g(x))
$$

yields a homotopy between them.

Lemma 5.1 Assume that $\operatorname{sing} u \cap \mathbf{B}_{1} \subseteq \mathbf{B}_{\varepsilon} L$ for some $0<\varepsilon<\frac{1}{5}$ and some $(n-3)$ dimensional plane $L$ through 0 . Assume further that u restricted to $L^{\perp} \cap \partial \mathbf{B}_{1 / 2}$ has homotopy type $\alpha$. Then

$$
L \cap \mathbf{B}_{1-\varepsilon} \subseteq \pi_{L}\left(\operatorname{sing}_{\geqslant \Theta} u \cap \mathbf{B}_{1}\right),
$$

where $\pi_{L}$ denotes the orthogonal projection onto L. In particular, $\operatorname{sing}_{\geqslant \Theta} u$ is $\varepsilon$-Reifenberg flat in $\mathbf{B}_{1}$.

Before giving the full proof, let us consider the special case when $\Theta(\alpha)$ is the lowest among all non-trivial homotopy types; in particular, this property holds if we consider maps 
into the standard sphere $\mathbb{S}^{2}$. In this case, the proof is simpler and does not depend on the deep results of Naber and Valtorta [7].

For each $y \in L \cap \mathbf{B}_{1-\varepsilon}, u$ restricted to the sphere $\left(y+L^{\perp}\right) \cap \partial \mathbf{B}_{\varepsilon}(y)$ has homotopy type $\alpha$, hence cannot be continuously extended to the ball $\left(y+L^{\perp}\right) \cap \mathbf{B}_{\varepsilon}(y)$. This shows the weaker inclusion $L \cap \mathbf{B}_{1-\varepsilon} \subseteq \pi_{L}\left(\operatorname{sing} u \cap \mathbf{B}_{1}\right)$. Recall that $\mathcal{H}^{n-3}$-a.e. point $z \in \operatorname{sing} u$ belongs to $\operatorname{sing}_{*} u$ and hence $\theta_{u}(z, 0) \geqslant \Theta$ due to our additional assumption. Since sing $\geqslant \Theta u$ is a closed set, we obtain the stronger inclusion.

Proof (of Lemma 5.1) For simplicity, let us assume $L=\mathbb{R}^{n-3} \times \mathbf{0}$ (by composing $u$ with a rotation, if necessary). Assume for the contrary that $L \cap \mathbf{B}_{1-\varepsilon}$ is not covered by the projection of sing $\geqslant \Theta u \cap \overline{\mathbf{B}}_{1-\varepsilon / 2}$. Since the latter is a compact set, it has to be disjoint with some small cylinder $\mathbf{B}_{\delta}^{n-3}(z) \times \mathbb{R}^{3}$ with $|z| \leqslant 1-\varepsilon$.

Recall that by the recent important work of Naber and Valtorta [7], the set $\operatorname{sing} u$ has finite $\mathcal{H}^{n-3}$ measure (locally, away from the boundary). Moreover, the set $\operatorname{sing} u$ is $(n-3)$ rectifiable and for $\mathcal{H}^{n-3}$-a.e. $y \in \operatorname{sing} u$ there exists an $(n-3)$-dimensional tangent plane Tan( $\operatorname{sing} u, y$ ) coinciding with $S(\varphi)$ for every tangent map $\varphi$ of $u$ at $y$. Let us temporarily assume that these tangent planes are transversal to $\mathbf{0} \times \mathbb{R}^{3}$, i.e.

$$
\operatorname{Tan}(\operatorname{sing} u, y) \pitchfork \mathbf{0} \times \mathbb{R}^{3} \text { for } \mathcal{H}^{n-3} \text {-a.e. } y \in \operatorname{sing} u \cap \mathbf{B}_{1-\varepsilon / 2} .
$$

We shall need Eilenberg's inequality, a Fubini-type inequality valid for any $\mathcal{H}^{n-3}$ measurable set $A$ with finite measure (see $[5,7.7,7.8]$ ):

$$
\int_{\mathbf{B}_{\delta}^{n-3}(z)} \mathcal{H}^{0}\left(A \cap \pi_{L}^{-1}(y)\right) \mathrm{d} y \leqslant \omega_{n-3} \mathcal{H}^{n-3}(A)
$$

Applying the above inequality twice-once with $A$ as the singular set and once with $A$ as its exceptional part of measure zero-we learn that for a.e. $y \in \mathbf{B}_{\delta}^{n-3}(z)$ the slice sing $u \cap \mathbf{B}_{1} \cap \pi_{L}^{-1}(y)$ consists of finitely many points, at each of them the tangent plane exists and is transverse to $\mathbf{0} \times \mathbb{R}^{3}$ (i.e. the direction of slicing).

Let us choose one such $y$ and denote these singular points by $p_{1}, \ldots, p_{k}$. Let also

$$
L_{j}:=\operatorname{Tan}\left(\operatorname{sing} u, p_{j}\right), \quad r_{0}:=\frac{1}{2} \min _{i \neq j}\left(\left|p_{i}-p_{j}\right|, \varepsilon-\left|p_{i}-y\right|\right) .
$$

For each $j=1, \ldots, k$, there is a $\operatorname{HCM} \varphi_{j}: \mathbb{R}^{n} \rightarrow \mathcal{N}$ with $S\left(\varphi_{j}\right)=L_{j}$ such that the sequence of rescaled maps $u_{r_{i}}(x)=u\left(p_{j}+r_{i} x\right)$ converges to $\varphi_{j}$ in $W_{\text {loc }}^{1,2}\left(\mathbb{R}^{n}\right)$ for some sequence $r_{i} \rightarrow 0$. Note that by our assumption, $\varphi_{j}$ has energy density strictly less than $\Theta$. Since this convergence is uniform away from $L_{j}$, maps $u_{r_{i}}$ and $\varphi_{j}$ are homotopic on $L_{j}^{\perp} \cap \partial \mathbf{B}_{1}$ for large enough $i$. Tilting $L_{j}^{\perp}$ to $\mathbf{0} \times \mathbb{R}^{3}$ and rescaling, we get that for some small $r_{j}<r_{0}$ the restriction of $u$ to $\pi_{L}^{-1}(y) \cap \partial \mathbf{B}_{r_{j}}\left(p_{j}\right)$ has the homotopy type of $\varphi_{j}$. Recalling that $u$ restricted to $\mathbf{0} \times \mathbb{R}^{3} \cap \partial \mathbf{B}_{1 / 2}$ (and hence also to $\pi_{L}^{-1}(y) \cap \partial \mathbf{B}_{\varepsilon}(y)$ ) has homotopy type $\alpha$, we conclude

$$
\alpha=\left[\varphi_{1}\right]+\cdots+\left[\varphi_{k}\right] \text { in } \pi_{2}(\mathcal{N}),
$$

where each $\varphi_{j}$ has energy density smaller than $\Theta$, which is a contradiction with the assumption that $\alpha$ is indecomposable.

To finish the proof, we need to get rid of the additional assumption (5.1). This is done by using the following simple transversality lemma. 
Lemma 5.2 Let $n=a+b$, consider the Grassmannian $G(n, a)$ with the standard Haar measure $\lambda$ and $G(n, b)$ with a finite positive Borel measure $\mu$. Then the set

$$
\{E \in G(n, a): \mu(\{F \in G(n, b): E \not h F\})>0\}
$$

has zero $\lambda$ measure.

Postponing its proof for the moment, we complete the reasoning as follows. Choose $a=3, b=n-3$, and let $\mu$ be the measure $\mathcal{H}^{n-3}\left\llcorner\operatorname{sing} u \cap \mathbf{B}_{1-\varepsilon / 2}\right.$ pushed-forward by the map $\operatorname{Tan}(\operatorname{sing} u, \cdot)$, i.e.

$$
\mu(U)=\mathcal{H}^{n-3}\left(\left\{y \in \operatorname{sing} u \cap \mathbf{B}_{1-\varepsilon / 2}: \operatorname{Tan}(\operatorname{sing} u, y) \in U\right\}\right) \text { for } U \subseteq G(n, n-3) .
$$

Then the set in Lemma 5.2 has measure zero, in particular its complement is dense. Hence we can choose $E \in G(n, 3)$ so that $E \pitchfork F$ for $\mu$-a.e. $F \in G(n, n-3)$, with $E$ arbitrarily close to $\mathbf{0} \times \mathbb{R}^{3}$. This amounts to satisfying (5.1) with a slightly tilted direction of slicing. Recall that sing $\geqslant \Theta u \cap \mathbf{B}_{1-\varepsilon / 2}$ is disjoint with the cylinder $\mathbf{B}_{\delta}^{n-3}(z) \times \mathbb{R}^{3}$, in consequence it is also disjoint with some smaller cylinder in direction $E^{\perp}$. It is easy to see that the rest of the proof remains unchanged.

Proof (of Lemma 5.2) First note that here $E \pitchfork F$ means these two linear subspaces intersect only at the origin. Thus, for each $F \in G(n, b)$ the set of all $E \in G(n, a)$ non-transversal to $F$ is a finite sum of smooth submanifolds of $G(n, a)$ of positive codimension

$$
\{E \in G(n, a): E \not F\}=\bigcup_{c=1}^{\min (a, b)}\{E \in G(n, a): \operatorname{dim} E \cap F=c\},
$$

hence it has zero $\lambda$ measure. Applying Fubini's theorem, we get

$$
\begin{aligned}
\int_{G(n, a)} \mu(\{F \in G(n, b): E \not h F\}) \mathrm{d} \lambda(E) & =\int_{G(n, a)} \int_{G(n, b)} \mathbf{1}_{E \not h F} \mathrm{~d} \mu(F) \mathrm{d} \lambda(E) \\
& =\int_{G(n, l)} \lambda(\{E \in G(n, a): E \not h\}) \mathrm{d} \mu(F) \\
& =0,
\end{aligned}
$$

so the integrand has to be zero for $\lambda$-a.e. $E \in G(n, a)$.

\subsection{Propagation of $\delta$-flatness to Finer Scales}

In this section we investigate some important consequences of Definition 4.3. Assuming that an energy minimizing map $u$ is $\delta$-flat in $\mathbf{B}_{1}$ (with small $\delta>0$ ), we shall see that $\operatorname{sing} u$ is actually more flat than a priori assumed (Lemma 5.3), $u$ is also $\delta$-flat in all smaller concentric balls (Corollary 5.5), and that $0 \in \operatorname{sing}_{\alpha} u$ (Corollary 5.6).

Lemma 5.3 For every $\varepsilon>0$ there is $\delta_{1}(\varepsilon)>0$ such that if $u$ is $\delta_{1}$-flat in $\mathbf{B}_{2}$, then we have $\|u-\varphi\|_{W^{1,2}\left(\mathbf{B}_{1}\right)} \leqslant \varepsilon$ for some HCM $\varphi$ of homotopy type $\alpha$ with energy density $\Theta$. Moreover, $\operatorname{sing} u$ is $\varepsilon$-Reifenberg flat in $\mathbf{B}_{1}$ with respect to the $(n-3)$-dimensional plane $S(\varphi)$.

Remark 5.4 For clarity, the conclusion above is stated on the twice smaller ball, but one can obtain a stronger conclusion $\left(\|u-\varphi\|_{W^{1,2}\left(\mathbf{B}_{2-\varepsilon}\right)} \leqslant \varepsilon\right.$ and $\varepsilon$-Reifenberg condition in $\left.\mathbf{B}_{1-\varepsilon}\right)$ by the same argument. However, restricting to a smaller ball is necessary due to the local nature of the $W^{1,2}$-compactness theorem, as well as possible singularities on $\partial \mathbf{B}_{2}$. 
Proof We employ the usual contradiction argument. Let $u_{k}$ be a sequence of minimizing harmonic maps such that $u_{k}$ is $1 / k$-flat in $\mathbf{B}_{2}$, with $\operatorname{sing} u \frac{1}{10}$-Reifenberg flat with respect to a fixed plane $L$. Choosing a subsequence, we have $u_{k} \rightarrow \varphi$ in $W_{\text {loc }}^{1,2}\left(\mathbf{B}_{2}\right)$ for some energy minimizing $\varphi$. By condition 1 in Definition 4.3, $\varphi$ is homogeneous with energy density $\Theta$. By Lemma 5.1, for each $k$ the set $\operatorname{sing} \geqslant \Theta u_{k}$ is $\frac{1}{10}$-Reifenberg flat in $\mathbf{B}_{1}$ with respect to $L$. Taking the limit and exploiting the upper semicontinuity of $\theta \cdot(\cdot, 0)$ with respect to both the map and the point, we conclude that the set

$$
S(\varphi) \equiv \operatorname{sing}_{\geqslant \Theta} \varphi
$$

is not contained in any $(n-4)$-dimensional plane. On the other hand, it is itself a linear subspace of dimension at most $n-3$, so we learn that $\varphi$ is a HCM of homotopy type $\alpha$; the homotopy property follows from uniform convergence away from $L$. For large enough $k, u_{k}$ is $\varepsilon$-close to $\varphi$ in $W^{1,2}\left(\mathbf{B}_{1}\right)$ and its singular set is contained in $\mathbf{B}_{\varepsilon} S(\varphi)$ (this is a consequence of upper semicontinuity of $\theta .(\cdot, 0)$ and $\varepsilon$-regularity $(2.1))$, which finishes the proof by another application of Lemma 5.1.

Corollary 5.5 If $\delta \leqslant \delta_{1}\left(\frac{1}{20}\right)$ and $u$ is $\delta$-flat in $\mathbf{B}_{2}$, then $u$ is also $\delta$-flat in any smaller ball $\mathbf{B}_{r}$ centered at 0 with $0<r \leqslant 1$.

Proof Condition 1 of Definition 4.3 is trivially satisfied. As for condition 2, it follows from Lemma 5.3 that sing $u$ is $\frac{1}{20}$-Reifenberg flat in $\mathbf{B}_{1}$, hence $\frac{1}{10}$-flat in any ball $\mathbf{B}_{r}$ with $\frac{1}{2} \leqslant r \leqslant 1$. In consequence, $u$ is $\delta$-flat in each of these balls. Then the claim follows by iteration of Lemma 5.3 rescaled to smaller and smaller balls.

Corollary 5.6 If $\delta \leqslant \delta_{1}\left(\frac{1}{20}\right)$ and $u$ is $\delta$-flat in $\mathbf{B}_{1}$, then every tangent map to $u$ at 0 is a HCM of type $\alpha$. In particular, $0 \in \operatorname{sing}_{\alpha} u$.

Proof Let $\varphi$ be any tangent map to $u$ at 0 , i.e. a $W_{\text {loc }}^{1,2}\left(\mathbb{R}^{n}\right)$-limit of rescaled functions $u_{k}(x)=$ $u\left(r_{k} x\right)$ for some sequence $r_{k} \rightarrow 0$; any thus obtained $\varphi$ is homogeneous. By Corollary 5.5, each $u_{k}$ is $\delta$-flat in $\mathbf{B}_{1}$ (with sing $u_{k} \frac{1}{10}$-Reifenberg flat with respect to some $L_{k}$ ), so the claim follows from Lemma 5.1 as in the proof of Lemma 5.3. The only difference is that the planes $L_{k}$ may change, but without loss of generality $L_{k} \rightarrow L$ in $G(n, n-3)$, which is enough to conclude that $\operatorname{sing}_{\geqslant \Theta} \varphi$ spans an $(n-3)$-dimensional plane.

\subsection{Moving the Ball Center}

Proposition 5.7 For every $\varepsilon>0$ there is $\delta_{2}(\varepsilon)>0$ such that if $u$ is $\delta_{2}$-flat in $\mathbf{B}_{2}$, then $u$ is $\delta_{1}(\varepsilon)$-flat in each of the balls $\mathbf{B}_{r}(z)$ with $z \in \operatorname{sing}_{\geqslant \Theta} u \cap \mathbf{B}_{1}$ and $0<r \leqslant 1 / 2$. Moreover, the sets $\operatorname{sing}_{\alpha} u$ and $\operatorname{sing}_{\geqslant \Theta} u$ restricted to the ball $\mathbf{B}_{1}$ coincide.

Proof Choose $\delta_{2}:=\min \left(\delta_{1}(\varepsilon), \delta_{1}(\eta / 2)\right)$ according to Lemma 5.3, where $\eta>0$ is to be fixed in a moment. Applying Lemma 5.3 rescaled to the ball $\mathbf{B}_{2}$, denote by $\varphi$ the approximating HCM and let $L=S(\varphi)$; according to Remark 5.4, we may assume the conclusion actually holds on the larger ball $\mathbf{B}_{3 / 2}$. To obtain the first claim, we first show that $\theta_{u}(z, 1 / 2) \leqslant$ $\Theta+\delta_{1}(\varepsilon)$ for each $z \in \mathbf{B}_{1} \cap \mathbf{B}_{\eta} L$. First,

$$
\int_{\mathbf{B}_{1 / 2}(z)}|\nabla u|^{2} \leqslant \int_{\mathbf{B}_{1 / 2}(z)}|\nabla \varphi|^{2}+C \eta^{2},
$$


by Lemma 5.3. If $z^{\prime}=\pi_{L}(z)$, then $\left|z-z^{\prime}\right|<\eta$ and

$$
\int_{\mathbf{B}_{1 / 2}(z)}|\nabla \varphi|^{2} \leqslant \int_{\mathbf{B}_{1 / 2+\eta}\left(z^{\prime}\right)}|\nabla \varphi|^{2}=(1+2 \eta)^{n-2} \Theta
$$

by $L$-invariance of $\varphi$ in $z^{\prime}$-direction. If $\eta$ is chosen small enough (depending on $\delta_{1}(\varepsilon)$ ), we obtain $\theta_{u}(z, 1 / 2) \leqslant \Theta+\delta_{1}(\varepsilon)$.

Since each point $z \in \operatorname{sing}_{\geqslant \Theta} u \cap \mathbf{B}_{1}$ lies in $\mathbf{B}_{\eta} L$ by Lemma 5.3, the above reasoning shows that condition 1 of Definition 4.3 holds for the ball $\mathbf{B}_{1 / 2}(z)$. Condition 2 is satisfied by our assumptions, so this ball is $\delta_{1}(\varepsilon)$-flat. Then Corollary 5.5 implies $\delta_{1}(\varepsilon)$-flatness of $u$ also in all smaller balls $\mathbf{B}_{r}(z)$ (to be precise, $\delta_{1}$-flatness for radii in the interval $\left(\frac{1}{4}, \frac{1}{2}\right)$ comes from the assumption of Reifenberg flatness).

By Corollary 5.6 we now have $z \in \operatorname{sing}_{\alpha} u$ for each $z \in \operatorname{sing}{ }_{\geqslant \Theta} \cap \mathbf{B}_{1}$. The inverse inclusion $\operatorname{sing}_{\alpha} u \subseteq \operatorname{sing}_{\geqslant \Theta} u$ is evident from the definition of $\Theta(\alpha)$.

Corollary 5.8 Under the assumptions of Proposition 5.7, the whole singular set sing $u$ restricted to the ball $\mathbf{B}_{1 / 2}$ coincides with $\operatorname{sing} \geqslant \Theta u$ (and hence with $\operatorname{sing}_{\alpha} u$ ).

Proof Assume that the ball $\mathbf{B}_{1 / 2}$ contains a point $p \in \operatorname{sing} u \backslash \operatorname{sing} \geqslant \Theta$. We may choose a point $z \in \operatorname{sing}_{\geqslant \Theta} u$ closest to $p$ (as it is a closed set) and set $r=2|p-z|$. Clearly $z \in \mathbf{B}_{1}$ and $0<r \leqslant 1$, so $u$ is $\delta_{1}(\varepsilon)$-flat in $\mathbf{B}_{r}(z)$. Choose $L=L(z, r)$ according to Definition 4.1. Then by Lemma 5.1 there is a point $z^{\prime} \in \operatorname{sing} \geqslant \Theta u \cap \mathbf{B}_{r}(z)$ such that $\pi_{L}\left(z^{\prime}\right)=\pi_{L}(p)$. Since both $\left|\pi_{L}(p)-p\right|$ and $\left|\pi_{L}\left(z^{\prime}\right)-z\right|$ are less than $\frac{r}{10}$, the triangle inequality yields a contradiction with minimality of $z$.

In order to apply the above results, one needs to know that $u$ is $\delta$-flat in at least one ball.

Lemma 5.9 Let $\delta>0$. If $0 \in \operatorname{sing}_{\alpha} u$ and $\theta_{u}(0,0)<\Theta+\delta$, then there is $r>0$ such that $u$ is $\delta$-flat in $\mathbf{B}_{r}$.

Proof Note that condition 1 of Definition 4.3 is trivially satisfied for small enough $r$.

By definition of $\operatorname{sing}_{\alpha} u$, some sequence of rescaled functions $u_{k}(x)=u\left(r_{k} x\right)$ converges in $W_{\text {loc }}^{1,2}\left(\mathbb{R}^{n}\right)$ to a $\mathrm{HCM} \varphi$ of homotopy type $\alpha$ for some sequence $r_{k} \rightarrow 0$. For large enough $k$, we have sing $u_{k} \cap \mathbf{B}_{1} \subseteq \mathbf{B}_{1 / 10} S(\varphi)$. Since the convergence is uniform away from $S(\varphi), u_{k}$ restricted to $S(\varphi)^{\perp} \cap \partial \mathbf{B}_{1 / 2}$ has homotopy type $\alpha$, so condition 2 follows from Lemma 5.1. Rescaling, we see that $u$ is $\delta$-flat in $\mathbf{B}_{r_{k}}$ for large enough $k$.

Remark 5.10 Combining Lemma 5.9 with Corollary 5.6, we see that some can be changed to any in the definition of $\operatorname{sing}_{\alpha}$, if only we restrict ourselves to points with energy density close to optimal. That is, if $y \in \operatorname{sing}_{\alpha} u$ and $\theta_{u}(y, 0)<\Theta+\delta_{1}\left(\frac{1}{20}\right)$, then every tangent map of $u$ at $y$ is a HCM of type $\alpha$.

We are now ready to prove the main theorem.

Proof (of Theorem 1.3) Fix the Hölder exponent $0<\gamma<1$ and choose $\varepsilon=\varepsilon(\gamma, n)>0$ according to Reifenberg's topological disc theorem (Theorem 4.2), then fix $\delta$ to be $\delta_{2}(\varepsilon)$ from Proposition 5.7.

Choose a point $p \in \operatorname{sing}_{\alpha} u$ such that $\theta_{u}(p, 0)<\Theta+\delta$. According to Lemma 5.9, $u$ is $\delta_{2}(\varepsilon)$-flat in some ball $\mathbf{B}_{2 r}(p)$. By Proposition 5.7, the set $\operatorname{sing}_{\alpha} u \cap \mathbf{B}_{r}(p)$ is closed and $\varepsilon$-flat in each ball $\mathbf{B}_{s}(z)$ centered at $z \in \operatorname{sing}_{\alpha} u \cap \mathbf{B}_{r}(p)$ with radius $0<s<r / 2$. Applying Theorem 4.2, we conclude that $\operatorname{sing}_{\alpha} u \cap \mathbf{B}_{r}(p)$ is bi-Hölder equivalent (with exponent $\gamma$ ) to an $(n-3)$-dimensional ball. 
By upper semicontinuity of $\theta_{u}(\cdot, 0)$ we can ensure $\theta_{u}(y, 0)<\Theta+\delta$ for all $y \in \mathbf{B}_{r}(p)$ (just by taking $r$ small enough), which together with Corollary 5.8 shows that the set in question forms an open subset of $\operatorname{sing} u$.

\section{Additional Results}

In this subsection we discuss two elementary observations that give a better description of $\delta$-flatness, but were not needed earlier in the proof of Theorem 1.3. We fix an indecomposable homotopy type $\alpha \in \pi_{2}(\mathcal{N})$ and its lowest energy level $\Theta=\Theta(\alpha)$.

The following lemma shows that condition 2 in Definition 4.3 can be dropped if one assumes a priori that $x \in \operatorname{sing}_{\alpha} u$. This gives us an equivalent condition for $\delta$-flatness.

Lemma 6.1 Assume that $0 \in \operatorname{sing}_{\alpha} u$. If $\delta \leqslant \delta_{1}\left(\frac{1}{20}\right)$ and $\theta_{u}(0,2) \leqslant \Theta+\delta$, then $u$ is $\delta$-flat in $\mathbf{B}_{1}$.

Proof Inspecting the proof of Lemma 5.3, we see that condition 2 of Definition 4.3 was only needed to ensure required symmetry of approximating homogeneous minimizer $\varphi$. Hence it would be enough to assume condition 2 of Definition 4.3 in a smaller ball $\mathbf{B}_{1 / 2}$, and $\delta$-flatness in $\mathbf{B}_{1}$ follows as in Lemma 5.3.

By Lemma 5.9, there is $r>0$ (possibly very small) such that $u$ is $\delta$-flat in $\mathbf{B}_{r}$. Applying the reasoning above, we see it is also $\delta$-flat in every ball $\mathbf{B}_{s}$ with $r \leqslant s \leqslant \min (1,2 r)$. An iteration of this argument (as in Corollary 5.5, but in the opposite direction) leads to the claim.

The last lemma gives a uniform bound (independent of $u$ ) for the rate of convergence $\theta_{u}(x, r) \rightarrow \theta_{u}(x, 0)$ when $r \rightarrow 0$, assuming $\theta_{u}(x, r)$ is already close to $\theta_{u}(x, 0)$. This assumption cannot be dropped, if only there exist tangent maps $\varphi: \mathbb{R}^{n} \rightarrow \mathcal{N}$ with $\operatorname{dim}_{H} \operatorname{sing} \varphi=n-3$ which are not HCMs.

An additional assumption is needed to ensure that the energy density is not greater than $\Theta$. This assumption is automatically satisfied if $\mathcal{N}$ is real-analytic or integrable in the sense of [17, Ch. 3.13]; see the remark preceding Corollary 1.4.

Lemma 6.2 Assume that $0 \in \operatorname{sing}_{\alpha} u$ and $\theta_{u}(0,1) \leqslant \Theta+\delta_{3}$ with $\delta_{3}(n, \alpha, \mathcal{N})>0$ sufficiently small, and assume additionally that $\Theta$ is an isolated energy level for HCMs of type $\alpha$. Then for every $\delta>0$ there is $r(\delta, n, \mathcal{N})>0$ such that $\theta_{u}(0, r) \leqslant \Theta+\delta$ (in consequence, $u$ is $\delta$-flat in $\mathbf{B}_{r}$ ).

Proof We choose $\delta_{3}>0$ smaller than $\delta_{1}\left(\frac{1}{20}\right)$ from Lemma 5.3 and such that

$$
\int_{\mathbf{B}_{1}^{n}}|\nabla \varphi|^{2} \notin\left(\Theta, \Theta+\delta_{3}\right]
$$

for each $\mathrm{HCM} \varphi$ of type $\alpha$.

For the sake of contradiction, assume there is a sequence of such energy minimizing maps $u_{k}$ with

$$
\Theta+\delta \leqslant \theta_{u_{k}}(0,1 / k) \leqslant \theta_{u_{k}}(0,1) \leqslant \Theta+\delta_{3} .
$$

Taking a subsequence, we obtain a limit map $u$ such that

$$
\Theta+\delta \leqslant \theta_{u}(0,0) \leqslant \theta_{u}(0,1) \leqslant \Theta+\delta_{3} .
$$

It follows from Lemma 6.1 that each $u_{k}$ is $\delta_{1}\left(\frac{1}{20}\right)$-flat in $\mathbf{B}_{1 / 2}$, hence so is $u$ and by Corollary 5.6 we infer $0 \in \operatorname{sing}_{\alpha} u$. In particular the energy density $\theta_{u}(0,0)$ is either $\Theta$ or greater than $\Theta+\delta_{3}$, a contradiction. 
Acknowledgements The author would like to thank Maciej Borodzik for fruitful discussions and helpful suggestions, and the anonymous referee for his careful reading of the manuscript.

Open Access This article is licensed under a Creative Commons Attribution 4.0 International License, which permits use, sharing, adaptation, distribution and reproduction in any medium or format, as long as you give appropriate credit to the original author(s) and the source, provide a link to the Creative Commons licence, and indicate if changes were made. The images or other third party material in this article are included in the article's Creative Commons licence, unless indicated otherwise in a credit line to the material. If material is not included in the article's Creative Commons licence and your intended use is not permitted by statutory regulation or exceeds the permitted use, you will need to obtain permission directly from the copyright holder. To view a copy of this licence, visit http://creativecommons.org/licenses/by/4.0/.

\section{References}

1. Brezis, H., Coron, J.M., Lieb, E.H.: Harmonic maps with defects. Commun. Math. Phys. 107(4), 649-705 (1986). http://projecteuclid.org/getRecord?id=euclid.cmp/1104116234

2. Hardt, R., Lin, F.H.: The singular set of an energy minimizing map from $B^{4}$ to $S^{2}$. Manuscr. Math. 69(3), 275-289 (1990). https://doi.org/10.1007/BF02567926

3. Lin, F.H., Wang, C.Y.: Stable stationary harmonic maps to spheres. Acta Math. Sin. (Engl. Ser.) 22(2), 319-330 (2006). https://doi.org/10.1007/s10114-005-0673-7

4. Luckhaus, S.: Partial Hölder continuity for minima of certain energies among maps into a Riemannian manifold. Indiana Univ. Math. J. 37(2), 349-367 (1988). https://doi.org/10.1512/iumj.1988.37.37017

5. Mattila, P.: Geometry of Sets and Measures in Euclidean Spaces: Fractals and Rectifiability. Cambridge Studies in Advanced Mathematics, vol. 44. Cambridge University Press, Cambridge (1995). https://doi. org/10.1017/CBO9780511623813

6. Mazowiecka, K., Miśkiewicz, M., Schikorra, A.: On the size of the singular set of minimizing harmonic maps into the 2-sphere in dimension four and higher (2019). https://arxiv.org/abs/1902.03161

7. Naber, A., Valtorta, D.: Rectifiable-Reifenberg and the regularity of stationary and minimizing harmonic maps. Ann. Math. (2) 185(1), 131-227 (2017). https://doi.org/10.4007/annals.2017.185.1.3

8. Nakajima, T.: Singular points of harmonic maps from 4-dimensional domains into 3-spheres. Duke Math. J. 132(3), 531-543 (2006). https://doi.org/10.1215/S0012-7094-06-13235-0

9. Reifenberg, E.R.: Solution of the plateau problem for $m$-dimensional surfaces of varying topological type. Acta Math. 104, 1-92 (1960)

10. Sacks, J., Uhlenbeck, K.: The existence of minimal immersions of 2-spheres. Ann. Math. (2) 113(1), 1-24 (1981). https://doi.org/10.2307/1971131

11. Schoen, R., Uhlenbeck, K.: A regularity theory for harmonic maps. J. Differ. Geom. 17(2), 307-335 (1982). http://projecteuclid.org/euclid.jdg/1214436923

12. Schoen, R., Uhlenbeck, K.: Boundary regularity and the Dirichlet problem for harmonic maps. J. Differ. Geom. 18(2), 253-268 (1983). http://projecteuclid.org/getRecord?id=euclid.jdg/1214437663

13. Schoen, R., Uhlenbeck, K.: Correction to: "A regularity theory for harmonic maps". J. Differ. Geom. 18(2), 329 (1983). http://projecteuclid.org/getRecord?id=euclid.jdg/1214437667

14. Simon, L.: Reifenberg's Topological Disc Theorem. http://www.math.uni-tuebingen.de/ab/analysis/pub/ leon/reifenberg/reifenberg.html

15. Simon, L.: Cylindrical tangent cones and the singular set of minimal submanifolds. J. Differ. Geom. 38(3), 585-652 (1993). http://projecteuclid.org/euclid.jdg/1214454484

16. Simon, L.: Rectifiability of the singular set of energy minimizing maps. Calc. Var. Partial Differ. Equ. 3(1), 1-65 (1995). https://doi.org/10.1007/BF01190891

17. Simon, L.: Theorems on regularity and singularity of energy minimizing maps. Lectures in Mathematics ETH Zürich. Based on Lecture Notes by Norbert Hungerbühler. Birkhäuser Verlag, Basel (1996). https:// doi.org/10.1007/978-3-0348-9193-6

18. Struwe, M.: On the evolution of harmonic mappings of Riemannian surfaces. Comment. Math. Helv. 60(4), 558-581 (1985). https://doi.org/10.1007/BF02567432

Publisher's Note Springer Nature remains neutral with regard to jurisdictional claims in published maps and institutional affiliations. 